\title{
V-PADA: Vehicle-Platoon-Aware Data Access in VANETs
}

\author{
Yang Zhang, Student Member, IEEE, and Guohong Cao, Fellow, IEEE
}

\begin{abstract}
The high mobility of vehicles and the unreliable wireless communication significantly degrade the performance of data access in vehicular ad hoc networks (VANETs). To address this problem, we propose a novel vehicle-platoon-aware data access solution called V-PADA. In V-PADA, vehicles contribute part of their buffers to replicate data for others in the same platoon and share data with them. When a vehicle leaves the platoon, it prefetches interested data and transfers its buffered data to other vehicles in advance so that they can still access the data after it leaves. To achieve this goal, V-PADA consists of two components: First, a vehicle-platooning protocol is designed to identify platoon formation and predict platoon splits. We use stochastic time series analysis to detect platoon and mobility anomalies and further introduce a two-step split prediction method to reduce the false alarm rate due to road curves. Second, a data management component is designed to guide platoon members to replicate and prefetch the most suitable data so that both high data availability and low data access overhead can be achieved. Extensive simulation results show that V-PADA can effectively improve the data access performance in VANETs.
\end{abstract}

Index Terms-Data replication, platoon, vehicular ad hoc network (VANET).

\section{INTRODUCTION}

$\mathbf{T}$ HE proliferation of low-cost wireless connectivity, combined with the growth of distributed peer-to-peer cooperative systems, is transforming next-generation vehicular networks. Drivers and passengers inside moving vehicles will be able to obtain and share their interested data, such as MP3 music, news, and video clips [1]. However, due to the high vehicle mobility, the topology of vehicular ad hoc networks (VANETs) dynamically changes, and disconnections may frequently occur. When two vehicles are disconnected, they are not able to access data from each other. Thus, data availability in VANETs is lower than that in conventional wired networks.

Data replication has been widely used to reduce the effect of intermittent connectivity and improve data access performance in distributed systems [2]. By replicating frequently accessed data at clients, they can continue to access data locally or from the nearby nodes that have the replica, even if the network

Manuscript received July 6, 2010; revised November 17, 2010 and February 7, 2011; accepted April 12, 2011. Date of publication April 29, 2011; date of current version June 20, 2011. This work was supported in part by the U.S. National Science Foundation under Grant CNS-0721479 and in part by Network Science Collaborative Technology Alliance under Grant W911NF-092-0053. The review of this paper was coordinated by Prof. P. Langendoerfer.

The authors are with the Department of Computer Science and Engineering, The Pennsylvania State University, University Park, PA 16802 USA (e-mail: yangzhan@cse.psu.edu; gcao@cse.psu.edu).

Color versions of one or more of the figures in this paper are available online at http://ieeexplore.iee.org.

Digital Object Identifier 10.1109/TVT.2011.2148202

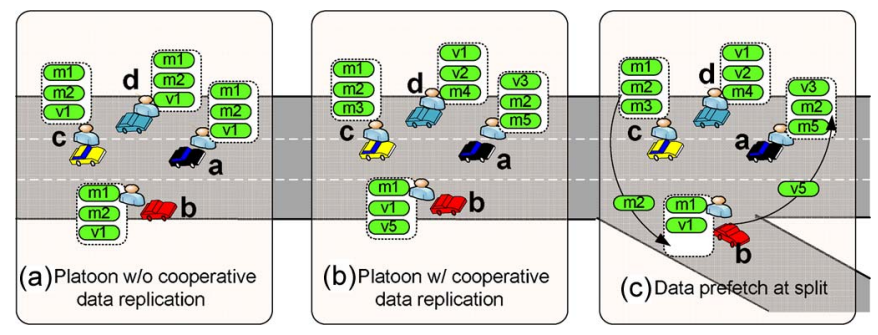

Fig. 1. Platoon-based data access in VANETs.

partitions. Generally speaking, data replication can increase data availability and reduce the query delay if there is plenty of storage space in the vehicles. However, many nodes may only have limited storage space, bandwidth, and power. For example, in a VANET, not only the vehicles but the drivers and passengers can be part of the network as well. Drivers and passengers want to share data with each other, but they may only have resource-constrained mobile phones, which have limited storage, and thus cannot replicate all the data such as large music files or video clips. In some vehicle-sensor integrated systems such as the MobEyes system [3], resource-constrained onboard sensor nodes only have limited storage, bandwidth, and power. To help vehicles and roadside infrastructure easily and quickly get the data, the data may have to be carefully placed. In addition, the contact time of vehicles may not always be long enough to transmit all data items. To replicate the data, nodes need to transmit it from other nodes, and obviously, there will be huge bandwidth and power cost for a large volume of data. By taking these issues into consideration, we expect that the nodes in a VANET, such as vehicles or in-vehicle mobile devices and sensors, should not be able (or willing) to replicate all data items in the network. Thus, we need to design new solutions for fast and convenient data access in VANETs.

Our solution is based on a well-known phenomenon called "vehicle platoon" [4] in VANETs, where vehicles often travel in closely spaced groups. A report from the Department of Transportation has indicated that the platooning probability for vehicles on highway can be higher than 70\% [5]. If vehicles move as a relatively stable platoon, they can contribute part of their buffer to replicate data for other vehicles in the same platoon and share data with them. Data redundancy in the same platoon can be reduced through cooperative replication; therefore, more data can be stored in the platoon, improving the data availability and reducing the data access delay. Fig. 1 shows an example of how platoon-aware data replication can be used to improve the performance of data access in a VANET. In this example, we assume that there are four vehicles $a, b, c$, 
and $d$ moving as a platoon. Each vehicle can hold three data items locally. In Fig. 1(a), vehicles have no knowledge of the existing platoon, and they replicate data based on their own interests. Therefore, if the top three interesting data items of four vehicles are the same, e.g., $m 1$ (music 1 ), $m 2$, and $v 1$ (video clip 1), all vehicles buffer the same data items. Obviously, in this case, there is too much data redundancy among these four vehicles, and only three data items are available to these vehicles. Fig. 1(b) shows how platoon-aware data access can help improve the performance. If vehicles know that they have formed a stable vehicle platoon, they can cooperatively replicate data and more efficiently organize their buffer. As the figure shows, instead of taking the same data items as vehicle $c$ does, vehicle $d$ may buffer data $v 2$ and $m 4$. Then, the data redundancy between vehicles $c$ and $d$ can be eliminated. However, some vehicles may leave the platoon, and they may not be able to access the data replicated by other platoon members. To address this problem, the splitting vehicle should prefetch its most interested data and transfer its buffered data copies to other platoon members. In Fig. 1(c), as vehicle $b$ leaves the platoon, it prefetches $m 2$ from vehicle $c$ and transfers $v 5$ to vehicle $a$. To achieve this goal, vehicles should be able to detect the split process as soon as possible, and then, they can prefetch and transfer data in advance before the split.

In this paper, we propose a novel vehicle-platoon-aware data access solution (called V-PADA) for VANETs, which includes two components. First, a vehicle-platooning protocol is proposed to quickly identify the platoon and predict the split process. In this protocol, stochastic time series analysis is used to detect vehicle platoon and mobility anomalies, and a two-step split prediction method is introduced to accelerate the detection and reduce false alarm due to road curvature. Second, a platoon-based data management component is introduced to achieve high data availability and reduce the intraplatoon data access cost. Specifically, we propose two cost-effective data replication algorithms to find the best vehicle to replicate each data item inside the platoon, and we provide data prefetch and transfer heuristics when there is a split detected. Extensive simulation results demonstrate that V-PADA can effectively improve the data access performance in VANETs.

The rest of this paper is organized as follows: The related work is summarized in Section II. Section III introduces the preliminaries of data access in VANET. Section IV describes the platooning protocol, which can quickly identify the platoon and predict the split process. In Section V, we present our data management solution. Performance evaluations are shown in Section VI. Finally, we conclude this paper in Section VII.

\section{RELATED WORK}

\section{A. Vehicular Networks}

Vehicular networks represent an interesting application scenario for not only traffic safety and efficiency but more commercial applications and entertainment support as well, such as service scheduling [6], [7], content sharing [1], [8], [9], peerto-peer marketing [10], and urban data collecting [11]. So far, however, most vehicular network researches have been focused on routing issues [12]-[15]. All these works assume that the data consumer is known beforehand so that the sender can always route the data to its destination. In this paper, we study a quite different data access paradigm in VANETs where each vehicle queries useful data from nearby neighboring vehicles. Our focus is to make use of the platoon mobility pattern of vehicles and cooperatively replicate data within platoon to improve the data access performance.

\section{B. Platoon Identification and Anomaly Detection}

Although vehicle platoons have not been used as a design parameter to facilitate data access in VANETs, their effects on traffic control and design of traffic signal timing have been studied for a long time. For example, a car-following model [16] was developed to describe vehicle platoon movements. Gaur and Mirchandani [17] and Jiang et al. [18] further developed algorithms to identify vehicle platoons from road traffic and optimized the setting of traffic signals. All these existing researches on vehicle platoon identification rely on roadside sensors for centralized vehicle mobility observation and analysis. Our work, however, investigates how to detect the platoon and the platoon partition by vehicles in a distributed manner without any centralized system support.

\section{Group-Based Data Access}

V-PADA optimizes data access by exploiting the vehicle platoon behavior, where vehicles often travel in closely spaced groups. There has been some work on group-based data access in mobile networks. Wang and Li [19] studied the service coverage problem in case of group partition. They assume that all nodes' mobility parameters are known beforehand to identify the group partition. Later, Huang and Chen [20] and Su and Zhang [21] investigated how to cooperatively share data or allocate channel within each mobile group. They both assume that groups are organized with explicit join/leave messages and that the partition is detected only after two nodes move out of their communication range. In V-PADA, split has to be predicted as soon as possible so that more data can be prefetched.

\section{Data Replication}

Data replication has been extensively studied in traditional distributed and database systems. However, in mobile networks, link failure frequently occurs, and data availability becomes an important issue. Hara [22], [23] proposed several data replication schemes for ad hoc networks. These schemes are based on the intuition that, to improve data availability, replicating the same data near neighboring nodes should be avoided. However, this intuition may not be valid when network partition is taken into consideration. Yin and Cao [24] and Fiore et al. [25] studied how to use data cache to increase data availability and reduce the data access cost, but their focus is not on mobility. V-PADA differs from the existing works in that it uses vehicle platoon to optimize data access. More importantly, V-PADA quickly predicts split and prefetches the necessary data. 


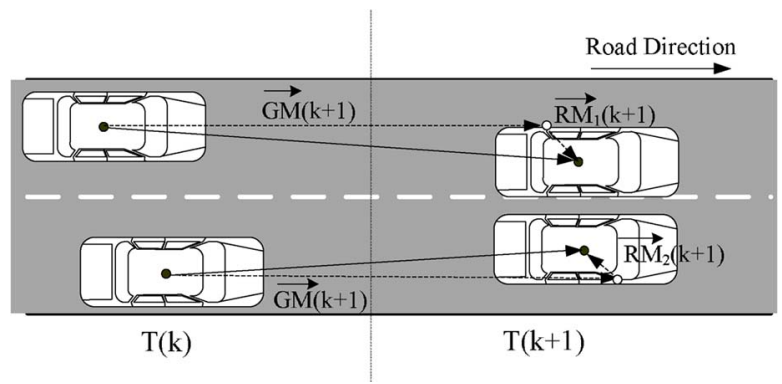

Fig. 2. Example of the platoon-based mobility model.

\section{PReliminaries}

\section{A. Data Access in VANET}

There are two different approaches for vehicles to access data in VANETs. The first approach relies on the support of roadside infrastructures. Each vehicle indirectly communicates with servers or other vehicles via base stations (e.g., 3G or WiMAX) or via access points (e.g., WiFi/802.11a,b,g, DSRC/802.11p). This approach is based on vehicle-to-vehicle communications, with which vehicles can communicate with their one-hop or multihop neighboring vehicles, exchanging and sharing interested information. Many studies have shown that the first approach is expensive and not convenient due to the high cost and the low bandwidth of the cellular communication [26], as well as the limited access opportunity and the infrastructure deployment constraint in the access-point-based communication [3], [27]. The vehicle-to-vehicle approach, however, is more flexible and cost effective in VANETs, particularly in rural or highway areas, which lack roadside infrastructure support. The vehicle-to-vehicle approach has been widely used in the literature [1], [12]-[14], [28] and will be used in this paper.

\section{B. Platoon-Based Mobility Model}

In VANETs, vehicles usually move as a platoon. Although there have been a few group mobility models such as the reference point group mobility (RPGM) [29] and its variations [19], they may not be directly applied to VANETs because they do not consider real-world constraints such as road layouts. In this paper, we combine road layout and speed limit with RPGM to model platoon-based VANET mobility. We assume that each vehicle platoon has a group motion vector $(\overrightarrow{G M})$ that defines the movement of the entire platoon. The group motion vector follows the road layouts. All vehicles in the same platoon share the same group motion vector and have different random motion vectors $(\overrightarrow{R M})$ due to their mobility deviation. Fig. 2 shows a movement example of two vehicles in the same platoon. The movement of each vehicle in each time slot is decided by the group motion vector and its own random motion vector. Supposing that the velocity of each vehicle follows a normal distribution $N\left(\mu, \delta^{2}\right)$, where $\mu$ is the average velocity and $\delta$ is the variance, because vehicles in the same platoon have the same average moving velocity and deviation, they share the same normal distribution parameter $\mu$ and $\delta$.
When a vehicle meets a platoon, it may join the platoon or stay alone. If the vehicle joins the platoon, it will follow the mobility pattern of the platoon with the corresponding velocity $N\left(\mu, \delta^{2}\right)$; otherwise, it keeps its own mobility pattern. Vehicles may leave the platoon when they 1) choose different routes at the road intersections or highway exits or 2) simply accelerate or decelerate. This platoon-based mobility model will be used in our later analysis and simulations.

\section{System Model}

There are $m$ vehicles in the network, which are denoted as $V_{i}(i=1 \ldots m)$, and each vehicle can store several data items in its buffer. There are $n$ data items, which are denoted as $D_{j}(j=1 \ldots n)$, and each data item $D_{j}$ has a size $S_{j}$. Each vehicle requests its interested data from time to time. The request frequency of vehicle $V_{i}$ to data $D_{j}$ is represented by $f_{i j}$. Note that the global access frequency of data $D_{j}$, i.e., $\sum_{i=1}^{m} f_{i j}$, represents the popularity of the data.

Fiore and Härri [30] showed that vehicles in the same platoon are relatively well connected. They are able to communicate with each other, either directly or through a small number of vehicle relays. Therefore, we assume that each platoon member knows the data replication arrangement within the platoon. Thus, after receiving a data request, the vehicle can easily locate the nearest platoon member that has the data. If none of the vehicles in the platoon has the data, the vehicle has to wait and check with any other encountered vehicle in the future. Each platoon has a "platoon leader," which can be selected based on different criteria and easily identified with the vehicles' periodic beacons [31], [32]. For example, it could be the vehicle that has stayed in the platoon for the longest time or the vehicle in the most front (or back) of the platoon. The main responsibility of the platoon leader is to maintain the data replication cycle (DRC) of the platoon and initiate the data-replicating process. At the beginning of each DRC, the "platoon leader" calculates the best replication arrangement based on some data replication algorithm and informs other platoon members to replicate data according to it.

We assume that all vehicles are equipped with communication devices and Global Positioning Systems (GPSs). With the communication devices, each vehicle has the ability to communicate with other vehicles within its communication range and is able to send and retrieve data content and its information regarding position to other vehicles via an ad hoc network. With the availability of a GPS system, it is practical for the vehicle to locate its position with certain accuracy so that vehicles can estimate not only their distance but the relative positions to each other as well. Here, we notice that there is another solution that uses the wireless signal strength of vehicles' beacon messages to estimate the distance between two vehicles. However, it is not able to identify the 2-D position. In the succeeding sections, we will indicate that, to provide fast and accurate mobility anomaly detection, not only the distance but the relative position change will be useful as well. Therefore, in this paper, we assume that the GPS device is a necessary equipment in our solution. 


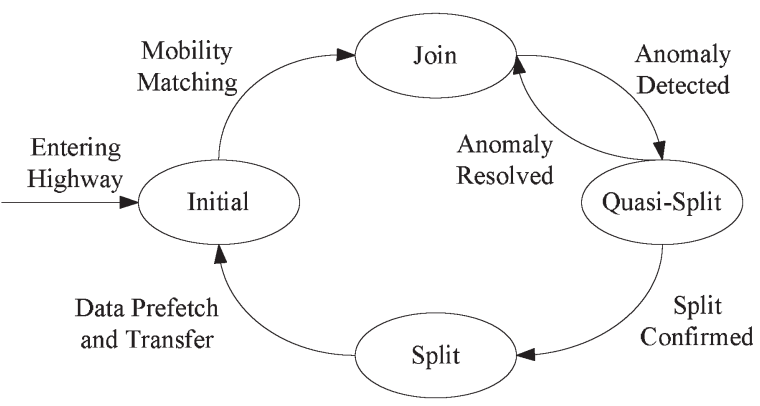

Fig. 3. State transition diagram of vehicles in V-PADA.

\section{Platooning Protocol}

\section{A. Protocol Overview}

The first component of V-PADA is the vehicle-platooning protocol, which is used to quickly identify the platoon and predict the split process. Fig. 3 uses a finite-state machine to describe the operating process of the platooning protocol.

At any given time, each vehicle stays at one of the following states: 1) Initial; 2) Join; 3) Quasi-Split; and 4) Split. When a vehicle enters the network, it is at the Initial state. Later, when it meets other vehicles in the same direction, it may join them as a platoon member. The join process can be detected with techniques that will be presented in Section IV-B. After one vehicle is detected to join the platoon, it enters the Join state and sends out a platoon-join message to all platoon members to announce that a new member has joined the platoon. The message contains the information of vehicle ID, its interest list, data list, and buffer size. As the platoon leader receives this message, it will use the information to determine the best data replication arrangement for the next replication cycle. When one vehicle detects mobility anomaly (more details in Section IV-C), it switches its state to Quasi-Split, where the anomaly will be further analyzed. If the anomaly comes from the change of road layout (e.g., the platoon is passing a curving road), the anomaly is resolved, and the vehicle returns to the Join state. Otherwise, if the vehicle is detected to split from the platoon, it enters the Split state. It sends out a platoon-split message to inform other platoon members that it is going to leave the platoon. At the same time, it starts to prefetch its interested data and transfer its buffered data to nearby platoon members. It is possible that messages may be lost for some reasons such as channel interference or collisions. However, this will not affect the performance of the platooning protocol too much because both join and split actions can always be detected by neighboring vehicles through its beacons. Furthermore, existing reliable and efficient broadcasting techniques [9] can be used to provide reliable message delivery.

Next, we discuss techniques used for platoon identification and split prediction in V-PADA.

\section{B. Stochastic Time Series Analysis for Platoon Identification}

In V-PADA, each vehicle maintains a Cartesian coordinate system, where the moving direction is the $X$-axis. Each vehicle (called monitoring vehicle) chooses the nearby vehicle as its

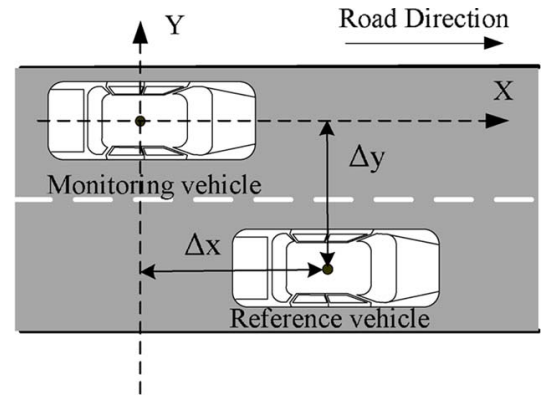

Fig. 4. Cartesian coordinate system.

reference vehicle (see more details in Section IV-C3). The coordinate of the reference vehicle is represented by its shortest distances to the $X$-axis and the $Y$-axis, which are denoted by $\Delta_{y}$ and $\Delta_{x}$, respectively. Fig. 4 gives an example of the coordinate system with one reference vehicle.

The monitoring vehicle and its reference vehicle periodically exchange their movement profile through beacon messages, ${ }^{1}$ by which the monitoring vehicle can get a series of relative coordinates of the reference vehicle in terms of $\Delta_{x}$ and $\Delta_{y}$. Then, by analyzing the $\Delta_{x}$ and $\Delta_{y}$ series, the monitoring vehicle can estimate the relative motion deviation between two vehicles and determine whether they are in the same platoon or not. If the motion deviation is consistently small, the monitoring vehicle can determine that it may have already joined the platoon.

During monitoring, the time interval between any two successive observations of $\Delta_{x}$ (or $\Delta_{y}$ ) is equal to the same beacon cycle; thus, the whole monitoring process can be regarded as a discrete and same-spaced process $\left\{X_{t}\right\}$ (and $\left\{Y_{t}\right\}$ for the $\Delta_{y}$ series). Therefore, in V-PADA, we can use the stochastic time series analysis on the observed position series to provide precise and automatic platoon identification. Specifically, we use real-time estimates of the variability of $\Delta_{x}$ and $\Delta_{y}$ as the forecasting thresholds. If one vehicle is in the Initial state and its relative position series of $\left\{X_{t}\right\}$ and $\left\{Y_{t}\right\}$ are within the range of the forecasting, the vehicle is considered to join the platoon. According to the time series analysis, by using the observation of $\Delta_{x}$ as an example, the whole identification process can be represented by a linear model

$$
\Phi_{p}(B) \nabla^{d}\left(X_{t}-\mu\right)=\theta_{q}(B) x_{t}
$$

where $p, d$, and $q$ are nonnegative integers; $\mu$ is the mean of the series; $\Phi_{p}(B)$ is an autoregressive operator of order $p ; \theta_{q}(B)$ is a moving average operator of order $q ; \nabla^{d}$ is a difference operator of order $d$; and $x_{t}$ 's are white noise variables independently distributed as $N\left(0, \delta_{x}^{2}\right)$. This model is usually referred to as an autoregressive integrated moving average (ARIMA) model of order $(p, d, q)$ [33].

The ARIMA model is fitted to an observed series by a threestage interactive procedure: 1) identification; 2) estimation; and

\footnotetext{
${ }^{1}$ The sampling frequency that each vehicle uses to monitor its own movement can be much higher than the frequency of beacon messages because there is no communication overhead involved. Therefore, the movement profile of each vehicle includes not only the latest moving direction and location information of the vehicle but the status of its moving direction as well, i.e., whether the moving direction is stable or continuously changes.
} 


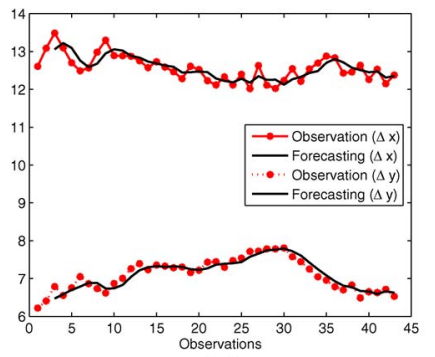

(a)

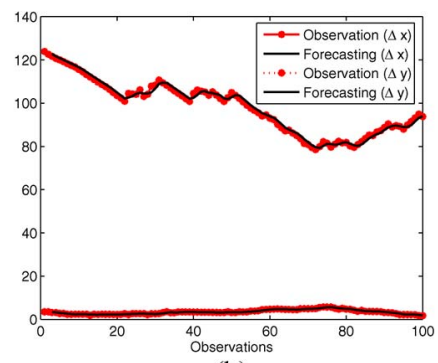

(b)
Fig. 5. Results of ARIMA model fitting with parameter $(0,1,3)$. (a) Berkeley Highway Laboratory data set (Vehicles 56 and 57). (b) Interstate 80 data set (Vehicles 4595 and 4709).

3) diagnostic checking. At the identification stage, the tentative model to fit the data is determined by inspecting the autocorrelation and partial autocorrelation functions of the series and its differences, and comparing them with those of some basic stochastic processes. At the estimation stage, maximumlikelihood estimates are obtained for the model parameters. The output observations are operated on by the inverse of the filter that is computed to have produced the white noise sequence $\left\{x_{t}\right\}$. Finally, the fitted model is diagnosed to ensure that the estimated model residuals are white noise deviations. Otherwise, the model should be redesigned by repeating the three stages of model construction.

We apply the described ARIMA model building procedure to several different mobility traces of vehicles moving on highways in Berkeley (Berkeley Highway Laboratory data set ${ }^{2}$ ) and Emeryville, CA (Interstate 80 data set $^{3}$ ). Our experiments with different values of $p, d$, and $q$ in the general class of models described by (1) show that ARIMA $(0,1,3)$ is refined enough to model the stochastic process (see Fig. 5), i.e., the first differences of $\Delta_{x}$ can be represented by a third-order moving average model of the form

$$
\nabla X_{t}=\left(1-\theta_{1} B-\theta_{2} B^{2}-\theta_{3} B^{3}\right) x_{t} .
$$

Then, the minimum mean-square error predictor of $X_{t+1}$, given the history of the process up to time $t$, which is denoted as $\widehat{X}_{t}$, is given by the conditional expectation

$$
\begin{aligned}
\widehat{X}_{t} & =E\left[X_{t+1} \mid\left(X_{t}, X_{t-1}, \ldots\right)\right] \\
& =X_{t}-\theta_{1} x_{t}-\theta_{2} x_{t-1}-\theta_{3} x_{t-2}
\end{aligned}
$$

and the forecast error made at time $t$ would be

$$
e_{t}=\widehat{X}_{t+1}-X_{t}=x_{t+1} \text {. }
$$

In other words, the random disturbances $\left\{x_{t}\right\}$ that generate the process are the succession of one-step-ahead forecast errors. Hence, the forecast made at time $t$ for $X_{t+1}$ can be expressed as

$$
\widehat{X}_{t}=X_{t}-\theta_{1} e_{t-1}-\theta_{2} e_{t-2}-\theta_{3} e_{t-3}
$$

\footnotetext{
${ }^{2}$ [Berkeley Data set] http://bhl.calccit.org:9006/bhl/

${ }^{3}$ [Interstate 80 Data set] http://ngsim.fhwa.dot.gov/
}

and the approximate $95 \%$ confidence interval limits for $X_{t+1}$ would be

$$
X_{t+1}( \pm)=\widehat{X}_{t} \pm 2 \delta_{x}
$$

where $\delta_{x}$ is the estimate of the standard deviation of the white noise variables, and it can be calculated by the existing location disturbance observations. Similarly, we can get the forecast limits with $99.7 \%$ and $68 \%$ confidence interval as $X_{t+1}^{99.7 \%}( \pm)=$ $\widehat{X}_{t} \pm 3 \delta_{x}$ and $X_{t+1}^{68 \%}( \pm)=\widehat{X}_{t} \pm \delta_{x}$, respectively. Then, the confidence limits for $Y_{t+1}$ can be similarly obtained. With the forecasting limits $X_{t+1}$ and $Y_{t+1}$, and the real-time observation series $\Delta_{x}$ and $\Delta_{y}$, each vehicle can decide whether it joins the platoon or not. If its position observations consistently stay within the forecasted position range, it should have joined the platoon; otherwise, it still moves alone.

Here, we note that, in V-PADA, one or multiple reference vehicles are used by the monitoring vehicle to estimate the relative movement stability of the vehicle and the platoon. An alternative solution is to use the baricentric position of the entire platoon as the reference instead of only taking a reference vehicle into account. However, to estimate the baricentric position of the platoon, the monitoring vehicle needs to get the position of all vehicles in the platoon, which requires all vehicles to flood their position information to all the platoon members. Obviously, to provide a good performance of position monitoring, the reference information needs to be quickly updated. However, highly frequent message flooding may bring in huge communication overhead and may even lead to network congestion within the platoon. Therefore, in V-PADA, we use the mobility of the reference vehicle to represent the mobility of the platoon. The relative position of the reference vehicle can be considered to be stable with the platoon if the reference vehicle detects no mobility anomaly of itself. Furthermore, each monitoring vehicle only needs to contact its neighboring reference vehicles to check the position change. Hence, there is no message flooding. In addition, we will show in Section IV-C3 that careful selection of the reference vehicle and multiple numbers of reference vehicles can improve the accuracy of anomaly detection.

\section{Split Prediction}

After joining a platoon, the monitoring vehicle keeps monitoring its reference vehicle and its own mobility pattern, so that it can quickly detect the split and have more time to prefetch and transfer data. In the following, we study how to detect the mobility anomaly and predict the vehicle split.

1) Anomaly Detection: The most intuitive approach in detecting mobility anomaly is only based on the distance between the monitoring vehicle and its reference vehicle. A mobility anomaly is detected when the distance becomes larger than a predefined threshold. However, it is difficult to find the appropriate threshold. If the threshold is large while the monitoring vehicle and the reference vehicle are close to each other, as shown in Fig. 6(a), the anomaly may not be detected, even after a relative large position change (from $\mathrm{A}$ to $\mathrm{A}^{\prime}$ ). If the distance threshold is small, it may result in high false positives, even if 


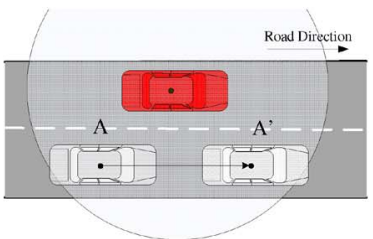

(a)

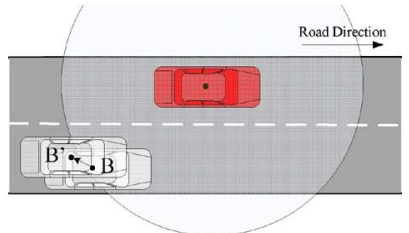

(b)

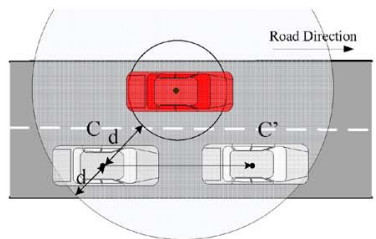

(c)

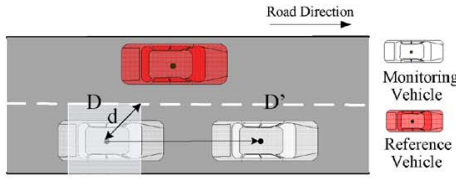

(d)

Fig. 6. Anomaly detection approaches. (a) and (b) Distance based (c) Distance change based (d) Position change based.

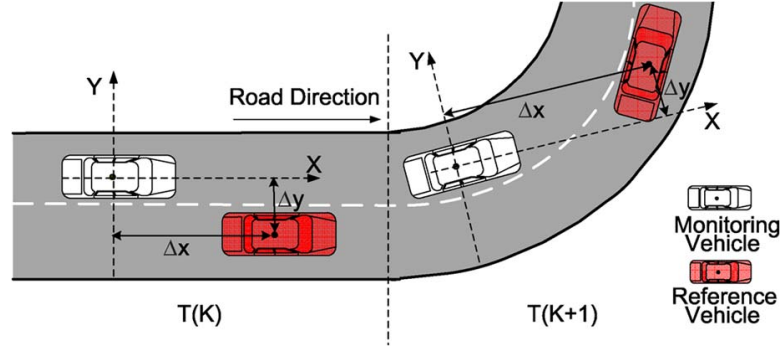

Fig. 7. Monitoring vehicle and its reference vehicle on a road curve.

the monitoring vehicle only moves a little bit [from B to B'; see Fig. 6(b)].

An alternative approach is based on the relative distance change. As shown in Fig. 6(c), suppose that the relative distance change threshold is $d$. An anomaly can be detected when the reference vehicle moves out of the shadow area, which is a torus with width $2 d$. However, in this case, some mobility anomalies may still not be detected, even if the position change between the two vehicles is much larger than the distance threshold (e.g., from $\mathrm{C}$ to $\mathrm{C}^{\prime}$, which usually happens when the vehicle suddenly accelerates to leave the platoon).

To address the weaknesses of the aforementioned approaches, we propose to use the 2-D relative position change between the monitoring vehicle and its reference vehicle to detect mobility anomaly. In this approach, we still use the Cartesian coordination system to determine the relative position of the reference vehicle in terms of $\Delta_{x}$ and $\Delta_{y}$ and use time series analysis on the relative position change to detect mobility anomaly. For example, as shown in Fig. 6(d), given the standard position deviation and the detection confidence interval, the detection boundary can be represented by a rectangle. If the diagonal of the rectangle is $2 d$, any mobility anomaly that results in a relative position deviation of distance $d$ can be detected. This approach can precisely determine the relative motion between the two vehicles and thus can be used to quickly detect any abnormal position change.

2) Two-Step Split Prediction: In the position-change-based approach, a large position change may come from the following reasons: 1) The vehicle is splitting from the platoon, or 2) the vehicle still stays with the platoon but the platoon changes its moving direction due to road layout such as a curve. As shown in Fig. 7, although the vehicle is in the same platoon as its reference vehicle, the observed relative position changes much in two successive observations due to the road curve, resulting in a false alarm of a vehicle split.

To mitigate the false alarm issue and quickly detect the split, we design a two-step split prediction method based on the following idea. If a vehicle moves on a straight road, its moving di- rection is usually stable; otherwise, if it is passing a curve road, its moving direction may continuously change. Furthermore, if a vehicle splits from the platoon, its distance to the reference vehicle increases as they move further apart; if the vehicle is still within the platoon, its distance from the reference vehicle may not change too much, even if they are moving on a curve road. By first analyzing the moving direction (the first step) and then comparing their relative distance deviation (the second step), we can differentiate different splitting scenarios and reduce the false alarm rate due to road curvature. In the following, we first categorize the possible splitting scenarios and then discuss how the two-step method works in different scenarios.

The vehicle split on a highway can be categorized into four scenarios.

1) Scenario I: The vehicle splits from the platoon by acceleration/deceleration [see Fig. 8(a)].

2) Scenario II: The vehicle splits from the platoon by entering a straight ramp [see Fig. 8(b)].

3) Scenario III: The vehicle splits from the platoon by entering a curving ramp [see Fig. 8(c)].

4) Scenario IV: The vehicle and the platoon split from each other by entering two different curving roads [see Fig. 8(d)].

In the first scenario, the mobility anomaly comes from the acceleration or deceleration of the splitting vehicle. Since vehicles move on the same straight road and in the same direction, this split can be easily confirmed in the first step by comparing their moving directions after the mobility anomaly is detected. If both the monitoring vehicle and its reference vehicle are in the same direction, scenario I is confirmed. In the second scenario, one of the vehicles (e.g., the reference vehicle) is still on the highway, but the other vehicle (e.g., the monitoring vehicle) changes its direction and moves onto a straight ramp. This split can be confirmed in the first step based on the vehicle moving direction, which is stable but different. Fig. 8(c) shows an example of scenario III, where the reference vehicle still moves on the highway, but the monitoring vehicle switches to a curving ramp. Because the moving direction of the monitoring vehicle keeps changing but the moving direction of the reference vehicle is stable, they are moving on different roads, and the split can be confirmed in the first step.

For scenario IV, as shown in Fig. 8(d), two vehicles move onto two different curve roads, and both vehicles continuously change their moving directions. However, it is possible that the two vehicles in the same platoon continuously change their moving directions on the same curve road. To determine whether they are moving on the same curve road or two different curve roads, we need to further analyze the distance deviation between them in the second step. If two vehicles pass 


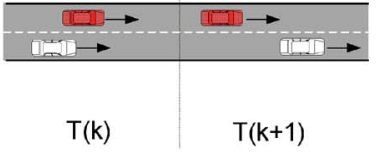

(a)

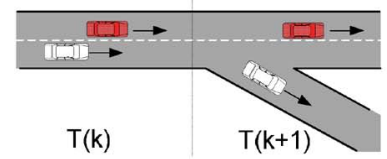

(b)

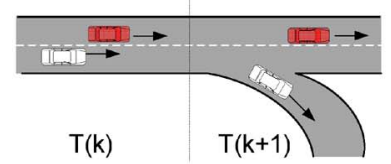

(c)

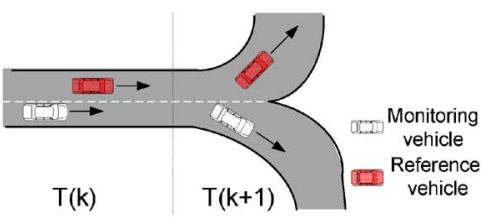

(d)

Fig. 8. Splitting scenarios.
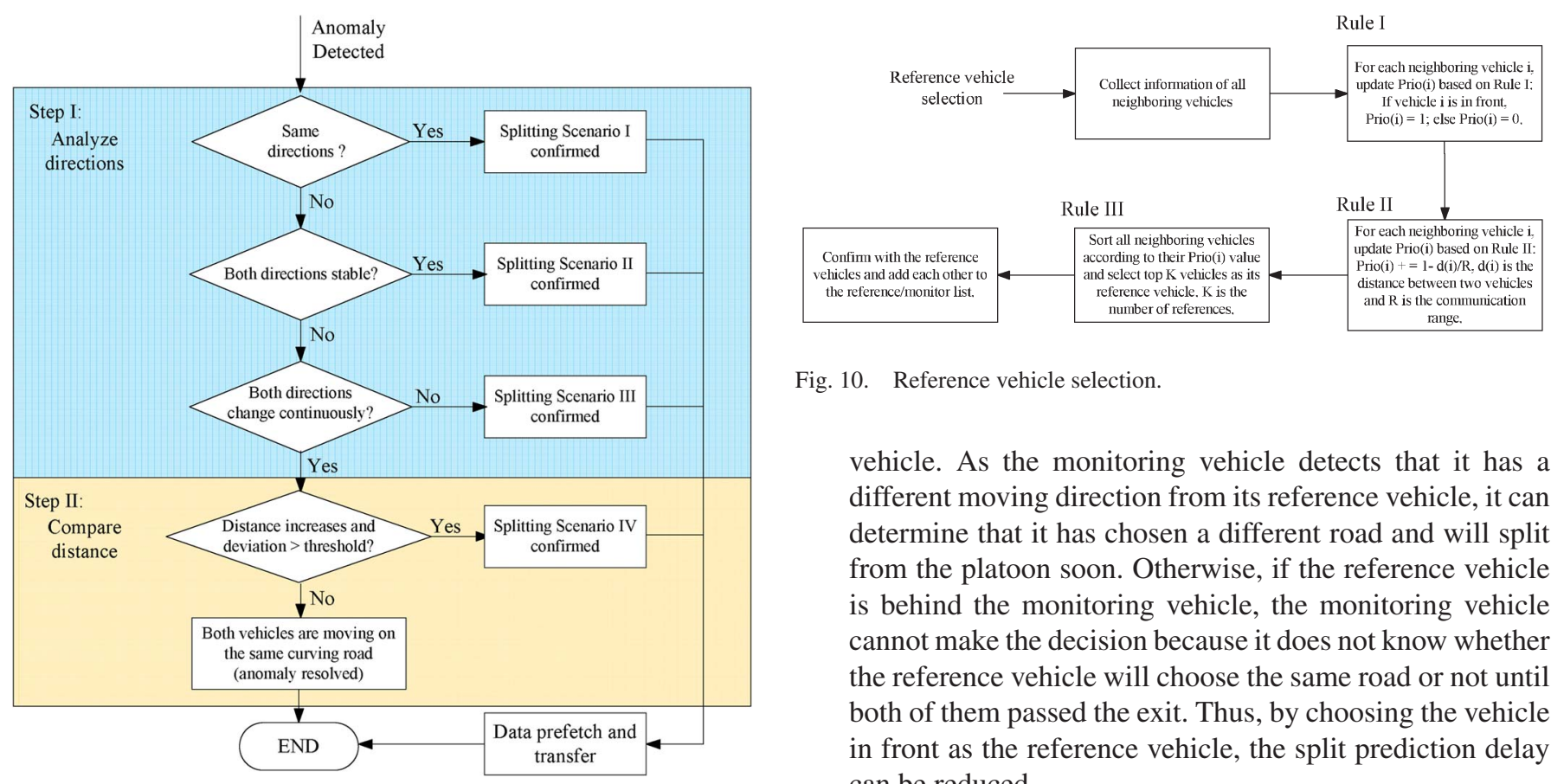

Fig. 10. Reference vehicle selection.

vehicle. As the monitoring vehicle detects that it has a different moving direction from its reference vehicle, it can determine that it has chosen a different road and will split from the platoon soon. Otherwise, if the reference vehicle is behind the monitoring vehicle, the monitoring vehicle cannot make the decision because it does not know whether the reference vehicle will choose the same road or not until both of them passed the exit. Thus, by choosing the vehicle in front as the reference vehicle, the split prediction delay can be reduced.

Fig. 9. Two-step split prediction method.

through the same curve road and there is no split, the distance between them should not change too much. However, if they move on two different curve roads, their distance increases when they move further away from each other. If the distance change between the two vehicle becomes larger than the threshold, which is called the splitting distance threshold, scenario IV can be confirmed.

The two-step split prediction method can be described by the flowchart shown in Fig. 9. As can be seen, most splits (scenarios I, II, and III) are confirmed in the first step by comparing the moving directions of two vehicles. The second step, which may bring in some prediction delay, is only applied when both the monitoring and reference vehicles move on the curve roads.

3) Reference Vehicle Selection: It is necessary to note that the relative position between the monitoring vehicle and its reference vehicle and the number of reference vehicles affect the result of anomaly detection and split prediction. For example, if the reference vehicle is far from the monitoring vehicle, any slight direction change of the monitoring vehicle may result in a large relative position deviation from the reference vehicle, which may result in prediction errors. To be more accurate, we have three rules for selecting the reference vehicle.

Rule I: A vehicle always chooses the vehicle in front of it (if any) as its reference vehicle. Rule I guarantees that the reference vehicle will pass the exit before the monitoring

Rule II: Each vehicle prefers the vehicle that is close to it as its reference vehicle. Rule II considers the communication reliability between the vehicle and its reference. If two vehicles are close to each other, they have more reliable communication and can more precisely determine their relative positions. Furthermore, a long distance between a vehicle and its reference vehicle may result in errors in the two-step split prediction. Therefore, the monitoring vehicle should choose the vehicle close to it as its reference vehicle.

Rule III: Multiple reference vehicles can improve the detection accuracy. If the monitoring vehicle has only one reference vehicle, any mobility anomaly from the reference vehicle will falsely alarm the monitoring vehicle, even though it is still within the platoon. Furthermore, it is possible that both the monitoring vehicle and the reference vehicle split from the platoon, and then, the monitoring vehicle cannot detect the split. Rule III helps reduce this kind of false positives and false negatives. By using multiple reference vehicles at the same time, a more accurate split prediction can be achieved.

In V-PADA, each vehicle selects its reference vehicles based on these three rules when it joins the platoon. When a reference vehicle is detected to split from the platoon, it will also apply these rules to find a new reference for its monitoring vehicle. The reference vehicle selection procedure is shown in Fig. 10, and the pseudocode is given in Algorithm 1. 


\section{Algorithm 1: Reference Vehicle Selection}

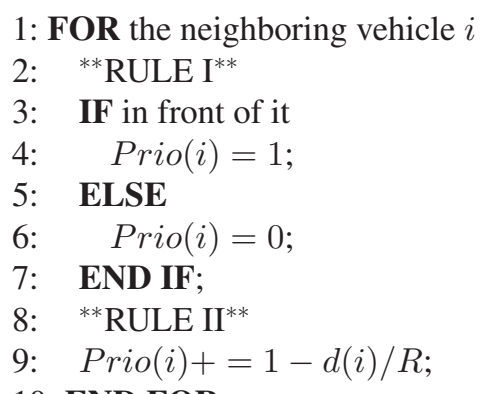

\section{0: END FOR}

\section{1: **RULE III**}

12: Sort vehicles according to Prio $(i)$;

13: WHILE $(K>0)$

14: $\quad$ Start from the vehicle $i$ with the highest $\operatorname{Prio}(i)$

15: DO

16: $\quad$ Add vehicle $i$ as its reference vehicle;

$$
\text { 17: } \quad K-- \text {; }
$$

18: END DO

19: END WHILE

\section{Platoon-Based Data Management}

In V-PADA, we exploit the platooning behavior to optimize the data access. First, we analyze the intraplatoon data replication problem and propose a cost-effective but centralized data replication algorithm called best-location data replication to help vehicles cooperatively access their interested data inside the platoon. The main purpose of the best-location replication algorithm is to optimally place data replicas at their best locations inside the platoon so that the vehicles in the same platoon can hold more interested data to avoid the long delay and low availability of accessing data not in the platoon. Second, we extend the best-location algorithm to a more scalable distributed algorithm called neighboring data replication, where each vehicle cooperatively replicates data with their directly connected neighboring nodes. Finally, we provide heuristics for vehicles to prefetch and transfer data before vehicle splits so that vehicles can still access their interested data after split.

\section{A. Intraplatoon Data Replication}

In V-PADA, data replication is periodically executed based on a predefined DRC that is maintained by the platoon leader. At the beginning of each DRC, the platoon leader calculates the best intraplatoon replication for each data item according to the data replication algorithm and then notifies all platoon members, with which each vehicle can buffer the most appropriate data replicas. In the following, we first formulate the intraplatoon data replication problem and then propose a new cost-effective replication algorithm that can remove data redundancy and reduce the data access cost.

1) Problem Formulation: We assume a vehicle platoon $G$ that consists of $l$ vehicles. A vehicle is denoted as $V_{i}$, where $i \in$
$[1 \ldots l]$. The buffer size of each vehicle is $M_{i}$, and the distance between any two vehicles $V_{i}$ and $V_{j}$ in terms of hops is $h_{i j}$. The set of data items available for access in the network is denoted as $D$. There are $d$ data items that are available in the platoon, each with data size $S_{i}$.

Let $P_{i}=\left\{p_{i j} \mid \forall D_{j} \in D\right\}$ represent the state of vehicle $V_{i}$ 's buffer, where

$$
p_{i j}= \begin{cases}1, & \text { if } D_{j} \text { is in the buffer of } V_{i} \\ 0, & \text { if } D_{j} \text { is not found in } V_{i} .\end{cases}
$$

The access probability of each vehicle is denoted as

$$
F=\left(\begin{array}{cccc}
f_{11} & f_{12} & \cdots & f_{1 d} \\
f_{21} & f_{22} & \cdots & f_{2 d} \\
\vdots & \vdots & \ddots & \vdots \\
f_{l 1} & f_{l 2} & \cdots & f_{l d}
\end{array}\right)
$$

We use $c_{i j k}$ to represent the cost for vehicle $V_{i}$ to access data $D_{j}$ from the vehicle in the same platoon, e.g., $V_{k}$. The access cost can be the access delay or the number of bytes to be transferred from $V_{k}$ or $V_{i}$. Then, $c_{i j k} \propto h_{i k} \cdot S_{j}$. For vehicle $V_{i}$, its total access cost can be calculated as

$$
\mathcal{C}_{i}=\sum_{j=1}^{d} f_{i j} \times \min \left\{c_{i j k} \mid \forall V_{k} \in G\right\} .
$$

Then, the goal of the cooperative replication algorithm is to find the best replication arrangement to optimize the following objective function:

$$
\min \left\{\sum_{i=1}^{l}\left(\mathcal{C}_{i}\right)\right\}
$$

subject to

$$
\sum_{j=1}^{d}\left(p_{i j} \times S_{j}\right) \leq M_{i} \quad \forall V_{i} \in G .
$$

The solution of (9) can be found through exhaustive search on all possible replication arrangements. However, let $\Lambda$ denote the set of all possible replication arrangements; the size of $\Lambda$ is equal to

$$
|\Lambda|=\sum_{i=1}^{l}\left(\begin{array}{c}
d \\
M_{i}
\end{array}\right)
$$

which is extremely large. The replication problem can be reduced to the metric uncapacitated facility location problem in the literature [34] that is known as NP-complete. Instead of trying to find a complex algorithm that is not practical to solve or approximate the problem, we propose a best-location data replication algorithm that can achieve near-optimal performance with small computation overhead.

2) Best-Location Data Replication Algorithm: The basic idea behind the best-location data replication algorithm is to find the best location (vehicle) to place each data replica so 
that the overall data redundancy and data access cost within the platoon are minimized. It is outlined here.

1) First, the average access probability of each data item within the platoon is calculated. The average access probability of a data item $D_{j}$ is defined as

$$
A_{j}=\frac{\sum_{i=1}^{l} f_{i j}}{l} .
$$

2) Let $v_{k j}$ denote the access cost if a copy of data $D_{j}$ is placed at vehicle $V_{k}$. Then, $v_{k j}$ is calculated as

$$
v_{k j}=\sum_{i=1}^{l} f_{i j} \times c_{i j k} .
$$

3) For each data item $D_{j}$, starting from that with the highest $A_{j}$, a vehicle $i$ is identified such that

$$
v_{i j}=\min \left\{v_{x j}\right\} \quad \forall \text { vehicle } V_{x} \in \text { Platoon. }
$$

4) The selected vehicle $V_{i}$ represents the best location to store $D_{j}$, which minimizes the total cost to access $D_{j}$ for the platoon. A copy of $D_{j}$ is placed at vehicle $V_{i}$, and this copy is called the primary copy of data $D_{j}$. If the buffer of vehicle $V_{i}$ is full, $D_{j}$ is given to the vehicle with the next lowest $v_{i j}$.

5) Steps 3 and 4 are repeated for all data items in the order of their average access probability until the buffers of all vehicles in the platoon are filled.

6) After all data items have been replicated once, the replication process starts again from the most frequently accessed to the least frequently access data. All data replications after the first round are called duplicated copy.

Formally, the best-location data replication algorithm is presented in Algorithm 2.

\section{Algorithm 2: Best-Location Data Replication Algorithm}

\section{1: Input:}

2: $\quad l$ : the number of vehicles in the platoon;

3: $d$ : the number of available data items in the platoon;

4: $f[][]$ : the 2-D matrix that records the access probability of each vehicle to each data item;

5: c[][][]: the 3-D matrix that records the access cost of each data item between any two vehicles in the platoon;

6:

7: Variables:

8: $A[]=0$ : the average access probability of each data item;

9: $v[][]=0$ : the total access cost of each data item for all vehicles in the platoon;

10 :

11: FOR each available data $D_{j}$

12: $\quad$ FOR each vehicle $V_{i}$

13: $\quad A[j]=A[j]+f_{i j}$;

14: $\quad$ FOR each vehicle $V_{k}$

15: $\quad v[k][j]=v[k][j]+f_{i j} \times c_{i j k}$;

16: $\quad$ END FOR

17: END FOR

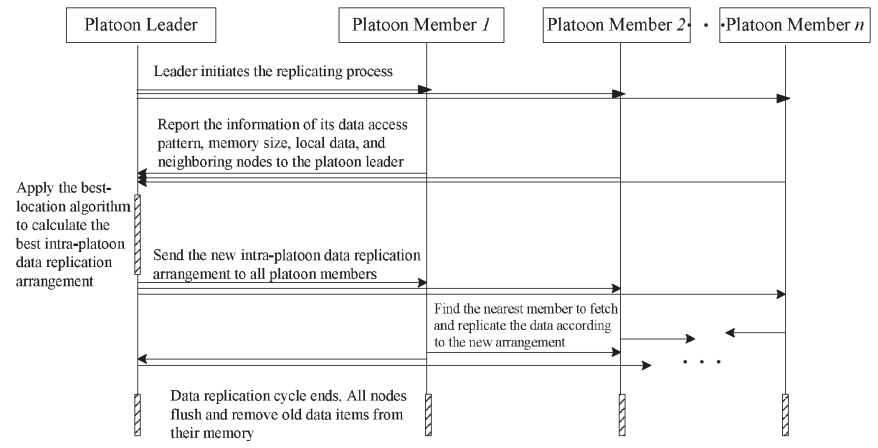

Fig. 11. Implementation of the best-location data replication.

\section{8: END FOR}

19:

20: WHILE there is memory available in the platoon

21: $\quad$ Start from the $D_{j}$ with the highest $A[j]$;

22: DO

23: $\quad$ Find vehicle $V_{i}$, s.t. $v[i][j]=\min \{v[x][j]\}$ for all $V_{x}$ in the platoon;

24: $\quad$ Make a copy of data $D_{i}$ at vehicle $V_{j}$;

25: $\quad$ Start from the data with the next highest $A[i]$;

26: END DO

27: END WHILE

Since no redundant replication takes place until every data item is replicated at least once, the best-location algorithm can guarantee that all available data have the opportunity to be buffered in the platoon. Furthermore, because the function for selecting the best vehicle to place each data replication [i.e., (14)] accounts for the access cost between the query vehicle and the nearest replication vehicle in the platoon, the best-location algorithm can also reduce the number of hops that the data need to be transferred to serve a query.

Implementation of the Best-Location Data Replication: The best-location algorithm requires several rounds of communications between the platoon leader and other platoon members and among vehicles that are involved in the data exchange. To more clearly make the entire data replication procedure, we list all the stages in the implementation of the best-location data replication algorithm in the flowchart (see Fig. 11).

As the figure shows, when a new DRC arrives, the platoon leader initiates the data-replicating process by sending a notification message to all platoon members. Upon receiving this message, all platoon members report the information of their data access patterns, their available buffer size, locally replicated data list, and their neighboring node lists to the platoon leader first. After the platoon leader receives all these information, it knows the topology of the platoon. It will calculate the best intraplatoon data replication arrangement according to the algorithm and send the new optimal arrangement to all platoon members in the second stage. When receiving the new arrangement, in the third stage, each platoon member fetches the data from the nearest node that has that data and makes a replication locally. Finally, in the last stage, all nodes in the platoon remove the old data items from the buffer, and thus, the newly released memory can be used by other applications. 
3) Neighboring Data Replication Algorithm: The bestlocation data replication algorithm can find the best vehicle to allocate each data item inside the vehicle platoon. However, this algorithm requires all platoon members to report their own information to the platoon leader. After the platoon leader collects all information, it calculates the best allocation of all data replications and notifies all platoon members. Obviously, this algorithm is centralized and may have large message overhead. To make the replication algorithm more scalable to large platoons, we propose a distributed data replication algorithm called neighboring data replication. In the neighboring replication algorithm, each vehicle only contacts with its directly connected neighbors, instead of all platoon members, and tries to find the best neighboring vehicle to replicate each data item and eliminate the data redundancy. More specifically, the algorithm is given here.

1) First, all vehicles are initially marked as "white," which means that no one has executed the allocation process yet.

2) Each vehicle starts the data replication process with some delay. The value of the delay depends on vehicle $i d$. For a smaller $i d$, the delay is shorter, and a longer delay is achieved for the vehicle with larger $i d$. Therefore, among all "white" vehicles, the vehicle with the smallest $i d$ will start the replication process first.

3) A vehicle sends an invitation to all its directly connected neighboring vehicles in the platoon, if it does not receive any invitation during its delay period. After receiving this invitation, if the neighboring vehicle is "white," it marks itself as "black" and sends back a message with its data access probability; otherwise, if the vehicle is already "black," it just discards the invitation.

4) The vehicle with the smallest $i d$ among its neighboring vehicles calculates the best allocation of all data replications for its neighbors, following the same data replication heuristic used in the best-location algorithm.

5) If all neighbors of a "white" vehicle are "black"; i.e., this "white" vehicle cannot find any neighbor to cooperate in the replication process, it only allocates its own most interested data items to its buffer.

Formally, the neighboring data replication algorithm is presented in Algorithm 3.

\section{Algorithm 3: Neighboring Data Replication Algorithm}

1: $\{$ begin to replicate data $\}$

2: all vehicles are marked as "white";

3:

4: IF vehicle $i$ is "white"

5: $\quad$ FOR all neighboring vehicles of $i$

6: IF it is "black"

7: do nothing and continue to the next vehicle;

8: ELSE

9: mark it as "black";

10: send back its data access probabilities and id;

11: $\quad$ END IF

12: END FOR
13: replicate all data items among neighboring vehicles following Algorithm 1;

\section{4: END IF}

The neighboring data replication algorithm eliminates the replica duplication among neighboring vehicles. Furthermore, it only requires the node to contact its neighboring vehicles to make the replication decision, which saves more communication overhead, compared with the best-location algorithm.

\section{B. Data Prefetch and Transfer on Splitting}

By cooperative replication, the two proposed data replication algorithms can eliminate intraplatoon data redundancy and then reduce the data access cost. However, the advantage of cooperative replication will be affected by platoon splitting. From the splitting vehicle point of view, it may not be able to access the most interested data placed at other platoon members after it is disconnected from the platoon. From the vehicle platoon point of view, if there are some primary data copies buffered at the splitting vehicle, the splitting may also significantly affect the intraplatoon data access. Moreover, if the primary data copy is the only data copy in the platoon, other platoon members will not be able to access the data after splitting.

To address this problem, the splitting vehicle should prefetch its most interested data and transfer its buffered primary data copies to other platoon members. More specifically, after a vehicle is confirmed as "split," it begins to immediately prefetch its interested data based on its own data access probability. Because all vehicles know the arrangement of data replications within the platoon, the splitting vehicle can easily locate the nearest nodes that have the data and prefetch it. To ensure that the split will not affect the data access of other platoon members, the splitting vehicle should transfer as many primary data copies as possible to its nearest neighbor that buffers duplicate copies of other data and replaces them with its primary data copies.

With data prefetch and transfer, the splitting vehicle can still locally access its interested data, and the negative effects on other platoon members can be minimized.

\section{Performance Evaluations}

In this section, we evaluate the performance of the proposed V-PADA and compare it with other solutions.

\section{A. Simulation Setup}

In our simulation, vehicles move within a fixed region of I79 (Interstate Highway) from Ohio River Blvd. to Clever $R d$. in a suburb of Pittsburgh, PA. It is a two-way highway and has three lanes in each direction. As shown in Fig. 12, there are three exits through which vehicles may leave the highway. To have a fixed number of vehicles in the simulation, we assume that the exit vehicle will enter the highway at the nearest highway end (End A or End B) and immediately start to move toward the opposite direction. Each vehicle in the simulation can initiate 


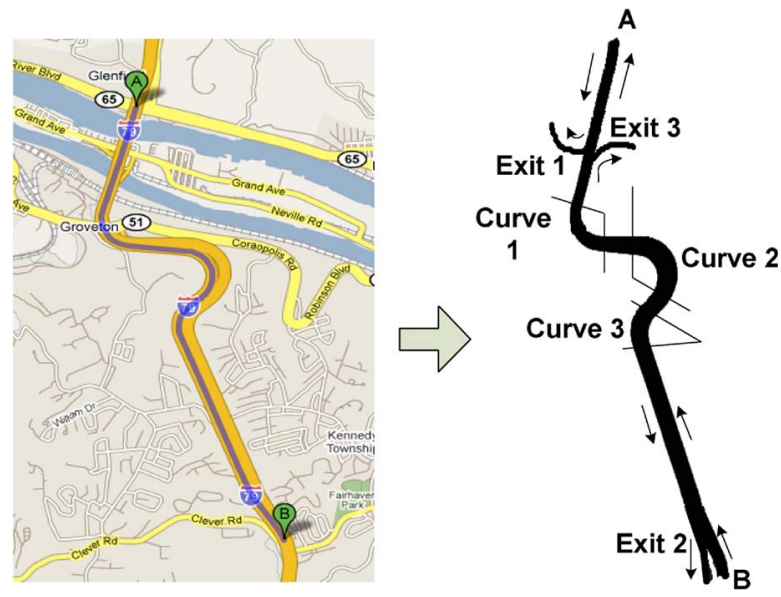

Fig. 12. Simulation setup (a 5-mi highway section of I79 in the Pittsburgh area).

queries for its interested data. If the query cannot be served by the vehicle but there is a copy of the requested data in the platoon, the request is sent to the nearest platoon member who has the data. If none of the vehicles in the platoon has the data, the request will be held until the vehicle meets another vehicle that has the data.

We implement V-PADA in the ns- 2 simulator ${ }^{4}$ with the CMU wireless extension. Since ns-2 is developed for generic ad hoc networks, it does not support VANET specific topologies and traffic control models. To provide a real VANET environment, we use the GrooveNet simulator ${ }^{5}$ and a map of the Pittsburgh $\operatorname{area}^{6}$ to generate a real highway topology. Then, we combine the highway topology with the platoon-based mobility model that is defined in Section III-B to generate the vehicle mobility trace file, which is used in the ns-2 simulations.

In the simulation, 180 vehicles move on the highway following the speed limits. There are six vehicle platoons, and each platoon has 15 members on average. One hundred different data items, with different data sizes $(5,15$, or $25 \mathrm{MB})$, are generated at the start of each simulation. Each vehicle can store up to $100-\mathrm{MB}$ data in its local buffer, but initially, it randomly picks data as its local data until the local buffer is full. The data access follows $Z i p f$ distribution, where the access probability of data $D_{i}$ is represented as $P_{i}=\left(1 / i^{\theta} \sum_{j=1}^{n}\left(1 / j^{\theta}\right)\right),(\theta \geq 0$, $n=100$ is the total number of data items in the network). Most of the system parameters and their default values are listed in Table I.

We compare V-PADA to three other solutions. The first solution, which is referred to as the noncooperative data access solution, does not consider the vehicle-platooning behavior, and each vehicle always caches its most interested data. The second solution, which is called connection-based cooperative

${ }^{4}[\mathrm{NS} 2]$ http://www.isi.edu/nsnam/ns

${ }^{5}$ [GrooveNet] http://www.seas.upenn.edu/ rahulm/Research/GrooveNet/ GrooveNet is a VANET simulator, which uses the map of the U.S. Census Bureau's TIGER/Line 2000+ database to generate real city/street level topologies and provides a variety of useful models for mobility, traffic control, etc., for VANET simulations. Therefore, we use GrooveNet to design the highway simulation scenario. We also rewrite the logger class of GrooveNet so that the logged mobility trace can be used in ns- 2 .

${ }^{6}$ [Tiger] http://www.census.gov/geo/www/tiger/
TABLE I

Simulation CONFIgURATIONS

\begin{tabular}{|l|c|}
\hline Parameter & Default Value \\
\hline Simulation Time & 20 minutes \\
\hline Number of Vehicles & 180 \\
\hline Number of Platoons & 6 \\
\hline Average Platoon Size & 15 vehicles \\
\hline Simulation Area & 5-mile highway on I79 \\
\hline Communication Range & $120 \mathrm{~m}$ \\
\hline Beacon Interval & $1.5 \mathrm{MB}, 4.5 \mathrm{MB}, 7.5 \mathrm{MB}$ \\
\hline Data Size & $24 \mathrm{Mbps}$ \\
\hline Data Transmission Rate & $30 \mathrm{MB}$ \\
\hline Memory Size & 100 \\
\hline Number of Data & 0.6 \\
\hline$Z$ ipf Parameter $\theta$ & $68 \%, \quad 95 \%, \quad 99.7 \%$ \\
\hline Detection Confidence & 5 minutes \\
\hline Data Replication Cycle & 1,3 \\
\hline Number of Reference Vehicles & HighwaySpeedModel \\
\hline Vehicle Speed Model & Highway speed $+15 \%$ \\
\hline Acceleration Speed & $\delta_{x}=3 m, \delta_{y}=2 m$ \\
\hline Platoon Mobility Parameter & \\
\hline
\end{tabular}

data access, makes use of vehicle platoon for cooperative data access. However, similar to most existing group-based data access solutions [20], [21], it does not have the anomaly detection and split prediction techniques used in V-PADA. The third solution, which is called the distance-based solution, extends the connection-based solution so that it can detect the vehicle split and prefetch data in advance. The split detection is distance based with a detection threshold of $80 \mathrm{~m}$.

The performance of these solutions are measured by the query delay and the data availability. The query delay is the average delay for a vehicle to receive the data after initiating a query. Data availability is the probability that the query can be successfully served before the vehicle moves out of the simulation area. Therefore, data availability is defined as

$$
\text { Data Availability }=\frac{\text { Number of successful queries }}{\text { Number of issued queries }} \text {. }
$$

Experiments were run using different workloads and system settings. The performance analysis presented here is designed to compare the effects of different workload parameters such as buffer size and Zipf parameter. For each workload parameter, the mean value of the measured data is obtained by collecting a large number of samples such that the confidence interval is reasonably small. In most cases, the $95 \%$ confidence interval for the measured data is less than $10 \%$ of the sample mean.

\section{B. Query Delay}

Fig. 13 compares V-PADA with noncooperative, connectionbased, and distance-based solutions in terms of query delay as a function of buffer size and data access skewness. Here, we note that we use V-PADA (best-location) to represent V-PADA with the best-location data replication algorithm and V-PADA (neighboring) to represent V-PADA with the neighboring data replication algorithm.

As shown in Fig. 13(a), when the buffer size is small (e.g., $15 \mathrm{MB}$ ), all schemes have relatively higher query delay. When the buffer size increases (e.g., to $450 \mathrm{MB}$ ), vehicles can buffer more data to serve the queries, and then, the query delay 


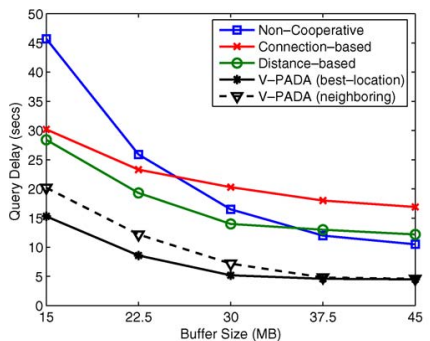

(a)

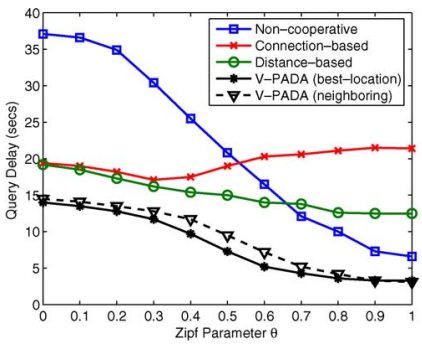

(b)
Fig. 13. Query delay. (a) Impact of buffer size. (b) Impact of data access skewness.

decreases. Fig. 13(a) also indicates that, when the buffer size is small (e.g., > $30 \mathrm{MB}$ ), the noncooperative solution has a much longer query delay than other solutions (e.g., up to $298 \%$ of V-PADA (best-location), 226\% of V-PADA (neighboring), $151 \%$ of the connection-based solution, and $160 \%$ of the distance-based solution). This confirms the fact that cooperative solutions are able to help improve the performance of data access. As shown in the figure, the smaller the buffer, the more the benefits that can be obtained from cooperative data access.

In our simulation, the performance of the noncooperative solution becomes better than the connection-based solution when the buffer size is larger than $30 \mathrm{MB}$. This is because, as the buffer size increases, more frequently accessed data can be locally buffered in the noncooperative solution. The connection-based solution, however, makes use of cooperation but does not have the split prediction capability. When split happens, the splitting vehicle does not have time to prefetch its interested data and transfer its data to other platoon members. Therefore, the splitting vehicle may not be able to access its most interested data after splitting, and the platoon may also lose some important data, which degrades data access performance. V-PADA (best-location) and V-PADA (neighboring) have the shortest query delay since they cooperatively buffer and organize data and they can quickly detect split and prefetch the necessary data. As a result, more query requests can be locally served even after disconnection, which further improves the data access performance compared with the distance-based solution. Here, we notice that V-PADA (best-location) achieves a bit better performance than V-PADA (neighboring). This is because the best-location data replication achieves platoonwide optimal data allocation, whereas the neighboring data replication algorithm only tries to eliminate data redundancy among neighboring vehicles.

Fig. 13(b) compares the query delay of different solutions as a function of data access skewness. In Zipf distribution, when $\theta=0$, the access pattern is uniformly distributed, and different data have similar popularity. As $\theta$ increases, the access pattern becomes more skewed. As can be seen from the figure, when the data access is close to uniform distribution, all the platoonaware data access solutions (V-PADA, connection based, and distance based) outperform the noncooperative solution, which is more sensitive to data access skewness. The noncooperative solution has lower query delay than the connection-based solution and the distance-based solution when data access becomes more skew (e.g., $\theta>0.6$ ), but V-PADA-both V-PADA (bestlocation) and V-PADA (neighboring) - consistently outper-

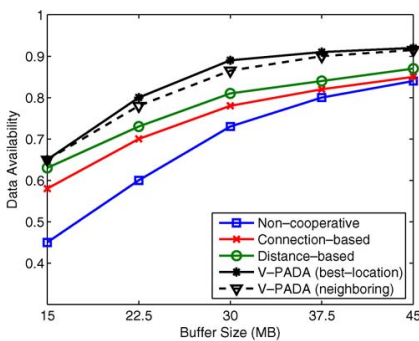

(a)

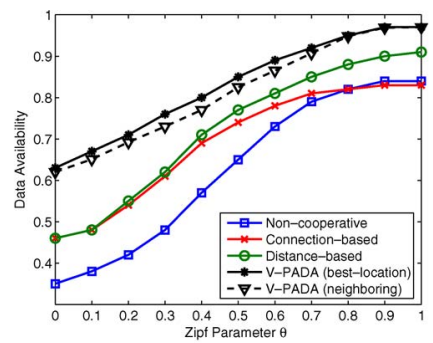

(b)
Fig. 14. Data availability. (a) Impact of buffer size. (b) Impact of data access skewness.

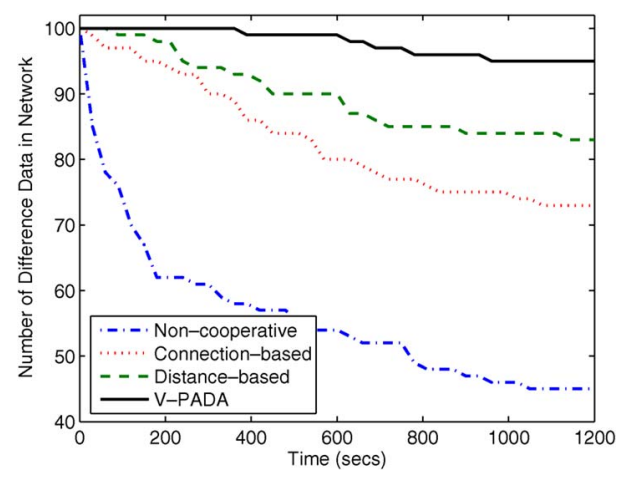

Fig. 15. Number of different data in the network at different simulation times.

forms the three other solutions. This confirms the importance of split detection and prefetch techniques used in V-PADA.

\section{Data Availability}

Fig. 14 evaluates different solutions on the data availability. Again, V-PADA (best-location) and V-PADA (neighboring) achieves much higher data availability than other solutions, i.e., up to $80 \%, 40 \%$, and $37 \%$ higher than the noncooperative solution, the connection-based solution, and the distance-based solution in Fig. 14(a), respectively. This is due to two reasons: 1) V-PADA cooperatively organizes data replications, either within the entire platoon (best-location) or among neighboring vehicles (neighboring); hence, V-PADA can hold more different data items than the noncooperative solution, which can be verified by results in Fig. 15. 2) V-PADA can quickly detect the split and prefetch the right data.

As shown in Figs. 13-15, although the noncooperative solution leads to the least number of different data items in the network, it still has similar or even better performance than the connection-based solution when data access becomes skew and buffer size becomes large. This is because the noncooperative solution prefers replicating the most frequently accessed data, thus having a relatively high local hit rate, compared with the connection-based solution, in which each vehicle may buffer data for other vehicles while does not have opportunity to prefetch its own interested data before splitting.

\section{Split Prediction}

In this section, we study the performance of different split prediction approaches, which are measured by the false positive 


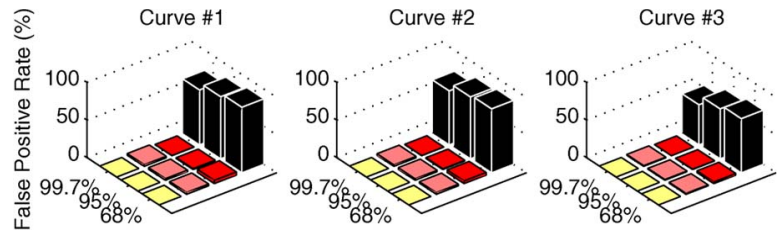

Fig. 16. Impact of detection confidence on the false positive rate.

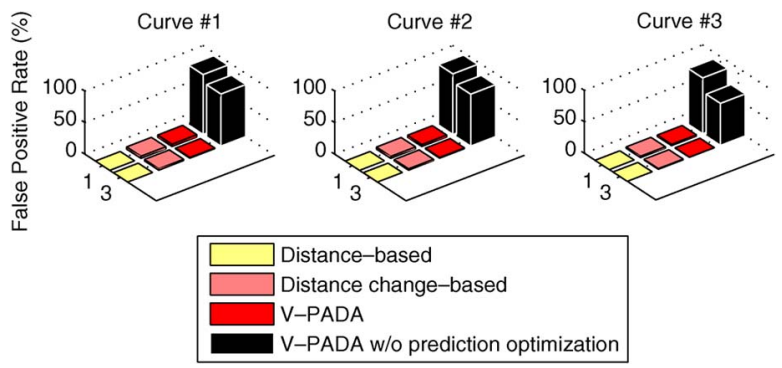

Fig. 17. Impact of the number of reference vehicles on the false positive rate

rate and the prefetch time. False positive may happen when a vehicle is still moving within the platoon but is falsely predicted to split. After a split is confirmed, the vehicle starts to prefetch data until it disconnects from the platoon. This time period is called the prefetch time. A long prefetch time indicates quick split prediction and, hence, more time for vehicles to prefetch and transfer data before splitting.

Because the noncooperative solution and the connectionbased solution do not have prediction capability, we only compare the proposed V-PADA with the distance- and distance-change-based solutions. The threshold of the distancechange-based solution is set to $15 \mathrm{~m}$. In addition, we add a fourth solution (which is denoted by $V-P A D A$ w/o prediction optimization) that uses the same time series analysis for anomaly detection as V-PADA but does not apply the two-step prediction method.

1) False Positive Rate: As shown in Figs. 16 and 17, V-PADA and the distance- and distance-change-based solutions have low false positive rates in all three curve sections, but the false positive rate of V-PADA without prediction optimization is very high. The low false positive rate of the distanceand distance-change-based solutions is because their distance thresholds for split prediction are larger than the relative position changes on the curve. V-PADA, however, is able to detect the mobility anomaly at curves and may treat it as a possible split in V-PADA without prediction optimization. This can be addressed by the two-step split prediction used in V-PADA, which can identify the curve and reduce the false positive. Figs. 16 and 17 also show that the false positive rate is related to the detection confidence interval and the number of reference vehicles. A higher detection confidence interval such as $99.7 \%$ or more numbers of reference vehicles can reduce the false positive rate.

By comparing the false positive rates at Curve 1, Curve 2, and Curve 3, we notice that the false positive rate at a sharp curve such as Curve 1 with a short radius is higher than that at a flat curve such as Curve 3 with a long radius. This is because

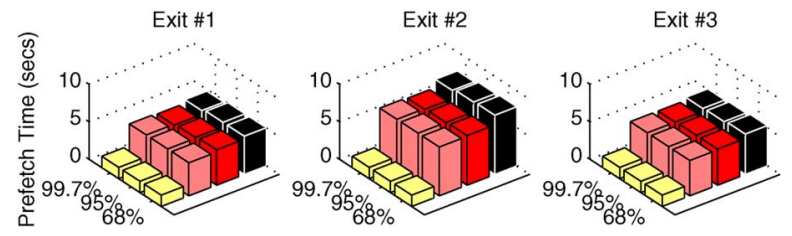

Fig. 18. Impact of detection confidence on the prefetch time.

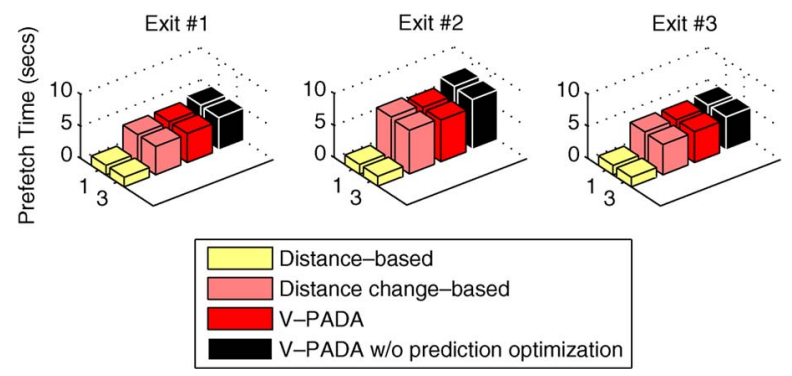

Fig. 19. Impact of the number of reference vehicles on the prefetch time.

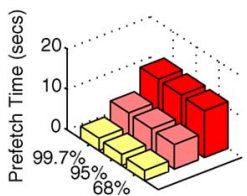

(a)

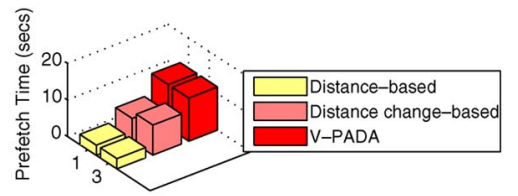

(b)
Fig. 20. Prefetch time when splitting due to acceleration. (a) With different detection confidence. (b) With different numbers of reference vehicles.

the sharp curve brings in a large position change, which leads to higher false positive.

2) Prefetch Time: Figs. 18 and 19 show the prefetch time of different solutions at different highway exits. From the figures, we can see that V-PADA has much longer prefetch time than the distance-based solution due to the use of time series analysis on the relative position deviations.

Fig. 20 shows the prefetch time in different solutions when vehicles accelerate to leave the vehicle platoon. Because there is no curve in this splitting scenario, we only compare the performance of V-PADA to the distance- and distance-change-based solutions. Again, V-PADA outperforms the other two, e.g., V-PADA detects splits up to $6 \mathrm{~s}$ faster than the distancechange-based solution and $9 \mathrm{~s}$ faster than the distance-based solution. This confirms the advantage of the position-changebased anomaly detection approach used in V-PADA that can quickly detect abnormal motion deviation.

The results of Figs. 18-20 also suggest that, in V-PADA, the impact of the detection confidence interval and the number of reference vehicles on the prefetch time is not big, compared with the impact on the false positive rate. This is because, as a split takes place, any mobility anomaly due to the split can always be quickly detected in V-PADA. The distance-changebased solution, instead, can take more advantage from multiple reference vehicles, because one single reference vehicle may neglect some large position deviation by only comparing the distance change, whereas multiple reference vehicles can reduce this kind of uncertainty. 


\section{CONCLUSION}

This paper has raised a challenging question: how to improve the performance of data access in an intermittently connected VANET. To answer this question, we have proposed V-PADA, which is a novel vehicle-platoon-aware data access solution for VANETs. V-PADA makes use of the "vehicle platoon" mobility pattern to collaboratively replicate data and optimize data access among vehicles. V-PADA consists of two components. First, a vehicle-platooning protocol is designed to identify the platoon and quickly predict vehicle split. Second, a data management component is introduced to achieve high data availability and reduce the intraplatoon data access cost. Simulation results have shown that V-PADA outperforms other data access solutions in VANETs.

To the best of our knowledge, this is the first work to exploit vehicle-platoon behavior for data access, considering various vehicle splits. The proposed solution in this paper is not limited to VANETs and can be extended to other mobile ad hoc networks. In the future, we will look into solutions for mobility anomaly detection in more complicated road structures and solutions for cooperative data access with the support of roadside infrastructures.

\section{REFERENCES}

[1] Y. Zhang, J. Zhao, and G. Cao, "Roadcast: A popularity aware content sharing scheme in VANETs," in Proc. IEEE ICDCS, 2009, pp. 223-230.

[2] Y. Huang, P. Sistla, and O. Wolfson, "Data replication for mobile computers," in Proc. ACM SIGMOD, 1994, pp. 13-24.

[3] U. Lee, E. Magistretti, M. Gerla, P. Bellavista, and A. Corradi, "Dissemination and harvesting of urban data using vehicular sensing platforms," IEEE Trans. Veh. Technol., vol. 58, no. 2, pp. 882-901, Feb. 2009

[4] D. Gerlough and M. Huber, "Traffic flow theory-A monograph," Transp. Res. Board, Washington, DC, Special Rep. 165, 1975.

[5] R. Hall and C. Chin, "Vehicle sorting for platoon formation: Impacts on highway entry and throughput," California PATH Program, ITS, Univ. California, Berkeley, Richmond, CA, California PATH Res. Rep. UCBITS-PRR-2002-7, 2002.

[6] Y. Zhang, J. Zhao, and G. Cao, "On scheduling vehicle-roadside data access," in Proc. ACM VANET, 2007, pp. 9-18.

[7] Y. Zhang, J. Zhao, and G. Cao, "Service scheduling of vehicle-roadside data access," Mobile Netw. Appl., vol. 15, no. 1, pp. 83-96, Feb. 2010.

[8] J. Rybicki, B. Scheuermann, M. Koegel, and M. Mauve, "Peertis: A peer-to-peer traffic information system," in Proc. ACM VANET, 2009, pp. 23-32.

[9] J. Zhao, Y. Zhang, and G. Cao, "Data pouring and buffering on the road: a new data dissemination paradigm for vehicular ad hoc networks," IEEE Trans. Veh. Technol., vol. 56, no. 6, pp. 3266-3277, Nov. 2007.

[10] U. Lee, J. Park, J. Yeh, G. Pau, and M. Gerla, "Code torrent: content distribution using network coding in VANET," in Proc. MobiShare, 2006, pp. 1-5.

[11] B. Hull, V. Bychkovsky, Y. Zhang, K. Chen, M. Goraczko, A. Miu, E. Shih, H. Balakrishnan, and S. Madden, "Cartel: A distributed mobile sensor computing system," in Proc. ACM SenSys, 2006, pp. 125-138.

[12] J. Zhao and G. Cao, "Vadd: Vehicle-assisted data delivery in vehicular ad hoc networks," IEEE Trans. Veh. Technol., vol. 57, no. 3, pp. 1910-1922, May 2008

[13] J. Jeong, S. Guo, Y. Gu, T. He, and D. Du, "TBD: Trajectory-based data forwarding for light-traffic vehicular networks," in Proc. IEEE ICDCS, 2009, pp. 231-238.

[14] A. Skordylis and N. Trigoni, "Delay-bounded routing in vehicular ad-hoc networks," in Proc. ACM MobiHoc, 2008, pp. 341-350.

[15] J. Burgess, B. Gallagher, D. Jensen, and B. Levine, "Maxprop: Routing for vehicle-based disruption-tolerant networks," in Proc. IEEE INFOCOM, 2006, pp. 1-11.

[16] R. Herman, E. Montroll, R. Potts, and R. Rothery, "Traffic dynamics: Analysis of stability in car-following," Oper. Res., vol. 7, no. 1, pp. 86106, Jan./Feb. 1959.
[17] A. Gaur and P. Mirchandani, "Method for real-time recognition of vehicle platoons," Transp. Res. Rec., vol. 1748, pp. 8-17, 2001.

[18] Y. Jiang, S. Li, and D. Shamo, "A platoon-based traffic signal timing algorithm for major-minor intersection types," Transp. Res., vol. 40, no. 7 , pp. 543-562, Aug. 2006.

[19] K. Wang and B. Li, "Efficient and guaranteed service coverage in partitionable mobile ad-hoc networks," in Proc. IEEE INFOCOM, 2002, pp. 1089-1098.

[20] J. Huang and M. Chen, "On the effect of group mobility to data replication in ad hoc networks," IEEE Trans. Mobile Comput., vol. 5, no. 5, pp. $492-$ 507, May 2006

[21] H. Su and X. Zhang, "Clustering-based multichannel MAC protocols for QoS provisionings over vehicular adhoc networks," IEEE Trans. Veh. Technol., vol. 56, no. 6, pp. 3309-3323, Nov. 2007.

[22] T. Hara, "Effective replica allocation in ad hoc networks for improving data accessibility," in Proc. IEEE INFOCOM, 2001, pp. 1568-1576.

[23] T. Hara and S. K. Madria, "Data replication for improving data accessibility in ad hoc networks," IEEE Trans. Mobile Comput., vol. 5, no. 11, pp. 1515-1532, Nov. 2006.

[24] L. Yin and G. Cao, "Supporting cooperative caching in ad hoc networks," IEEE Trans. Mobile Comput., vol. 5, no. 1, pp. 77-89, Jan. 2006.

[25] M. Fiore, F. Mininni, C. Casetti, C. Chiasserini, "To cache or not to cache?" in Proc. IEEE INFOCOM, 2009, pp. 235-243.

[26] J. Eriksson, H. Balakrishnan, and S. Madden, "Cabernet: vehicular content delivery using WiFi," in Proc. MobiCom, 2008, pp. 199-210.

[27] J. Zhao, T. Arnold, Y. Zhang, and G. Cao, "Extending drive-thru data access by vehicle-to-vehicle relay," in Proc. ACM VANET, 2008, pp. 66-75.

[28] D. Jiang, Q. Chen, and L. Delgrossi, "Optimal data rate selection for vehicle safety communications," in Proc. ACM VANET, 2008, pp. 30-38.

[29] X. Hong, M. Gerla, G. Pei, and C. Chiang, "A group mobility model for ad hoc wireless networks," in Proc. ACM MSWiM, 1999, pp. 53-60.

[30] M. Fiore and J. Härri, "The networking shape of vehicular mobility," in Proc. ACM MobiHoc, 2008, pp. 261-272.

[31] M. Abuelela, S. Olariu, and I. Stojmenovic, "Opera: Opportunistic packet relaying in disconnected vehicular ad hoc networks," in Proc. IEEE MASS, 2008, pp. 285-294.

[32] F. Bai and B. Krishnamachari, "Spatio-temporal variations of vehicle traffic in VANETs: Facts and implications," in Proc. ACM VANET, 2009, pp. $43-52$.

[33] P. J. Brockwell and R. A. Davis, Introduction to Time Series and Forecasting. New York: Springer-Verlag, 1996.

[34] T. H. Cormen, C. E. Leiserson, R. L. Rivest, and C. Stein, Introduction to Algorithms, 2nd ed. Cambridge, MA: MIT Press, 2001.

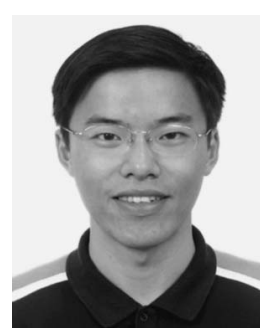

Yang Zhang (S'05) received the B.S. and M.E. degrees from Nanjing University, Nanjing, China, in 2002 and 2005, respectively. $\mathrm{He}$ is currently working toward the Ph.D. degree with the Department of Computer Science and Engineering, The Pennsylvania State University, University Park.

His research interests include distributed systems, mobile computing, and vehicular ad hoc networks.

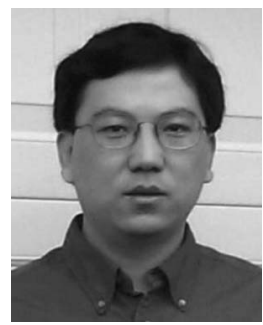

Guohong Cao (F'11) received the B.S. degree from Xi'an Jiaotong University, Xi'an, China, and the M.S. and Ph.D. degrees in computer science from Ohio State University, Columbus, in 1997 and 1999, respectively.

Since then, he has been with the Department of Computer Science and Engineering, The Pennsylvania State University, University Park, where he is currently a Professor. He has published more than 150 papers on wireless sensor networks, wireless network security, vehicular ad hoc networks, data access and dissemination, and distributed fault-tolerant computing. His research interests are wireless networks and mobile computing.

Dr. Cao has served on the Editorial Board of the IEEE TRANSACTIONS ON Mobile Computing, the IEEE TRANSACTIONS ON WiRELESS CoMmuniCATIONS, and IEEE TRANSACTIONS ON VEHICULAR TECHNOLOGY, and as program committee member of many conferences. He was the recipient of the National Science Foundation CAREER Award in 2001. 